\title{
General no-go condition for stochastic pumping
}

\author{
Christian Maes, ${ }^{1, a)}$ Karel Netočný, ${ }^{2, b)}$ and Simi R. Thomas ${ }^{1}$ \\ ${ }^{1}$ Instituut voor Theoretische Fysica, K.U. Leuven, B-3001 Leuven, Belgium \\ ${ }^{2}$ Institute of Physics AS CR, Prague CZ-18221, Czech Republic
}

(Received 19 February 2010; accepted 14 May 2010; published online 21 June 2010)

\begin{abstract}
The control of chemical dynamics requires understanding the effect of time-dependent transition rates between states of chemomechanical molecular configurations. Pumping refers to generating a net current, e.g., per period in the time dependence, through a cycle of consecutive states. The work of artificial machines or synthesized molecular motors depends on it. In this paper we give short and simple proofs of no-go theorems, some of which appeared before but here with essential extensions to non-Markovian dynamics, including the study of the diffusion limit. It allows to exclude certain protocols in the working of chemical motors where only the depth of the energy well is changed in time and not the barrier height between pairs of states. We also show how pre-existing steady state currents are, in general, modified with a multiplicative factor when this time dependence is turned on. (C) 2010 American Institute of Physics. [doi:10.1063/1.3446811]
\end{abstract}

\section{INTRODUCTION}

Molecular cybernetics deals with the control and the steering of system of molecules. As part of systems chemistry, it investigates models of chemical networks with timedependent dynamics. A common application is found in the study of molecular motors or mesoscopic machines. Here, chemical or electrical gradients repetitively and progressively drive a system away from equilibrium in such a way that when the motor returns to its original configuration, a physical task performed by the machine is not undone. ${ }^{1,2}$ This technology is ubiquitous in nature in the form of translational and rotational movements, e.g., in muscle fibers, bacterial flagella and cilia, ${ }^{3,4}$ or to transport material within and across a cell membrane, either powered by solar energy (as in photosynthesis) or by chemical energy stored in molecular bonds (e.g., adenosine triphosphate).

Artificial designs analogous to such motors use external time-dependent perturbations such as light, heat, or chemical stimulus to drive the system. ${ }^{5-9}$ It is thus relevant, in attempts to synthesize and control artificial molecular motors, to understand the relation between the external pumping and creation of systematic flows. Previously, similar questions were asked by Thouless ${ }^{10}$ for electronic pumping. Astumian and Derény ${ }^{11}$ studied charge transfer from a lower to a higher chemical potential by varying the gate and portal energies. Astumian ${ }^{12}$ also analyzed the adiabatic regime of ion pumping in externally driven protein structures and in a molecular motor based on a three-ring catenane. ${ }^{13}$ A general theory of adiabatic pumps in terms of geometrical phase was proposed by Sinitsyn and Nemenman. ${ }^{14}$ Chernyak and Sinitsyn ${ }^{15}$ discovered that the adiabatic pumping currents become quantized at low temperatures. Generalizations beyond adiabatic regimes are so far limited to Markov models.

In the present paper we concentrate on no-go or no-

\footnotetext{
${ }^{a)}$ Electronic mail: itf.fys.kuleuven.be/ christ/.

${ }^{b)}$ Electronic mail: netocny @fzu.cz.
}

pumping theorems, stating the absence of a net timeaveraged current under certain protocols. We refer to the experiment of Leigh et al. ${ }^{5}$ on unidirectional motion in (2)- and (3)-catenanes. Rahav et al. ${ }^{16}$ were the first to give a nopumping theorem for jump processes with nonadiabatic pumping and generalization to diffusion processes. ${ }^{17}$ This was further studied and systematized by Chernyak and Sinitsyn ${ }^{18}$ so that the following general conclusion was reached: When the dynamics can be modeled as a Markov state system for which the transitions between states $x$ and $y$ have an Arrhenius-type time dependence

$$
w_{t}(x, y)=A(x, y) e^{G_{t}(x) / k_{B} T}, \quad A(x, y)=A(y, x),
$$

with periodic time-dependent energy wells $G_{t}(x)$ and with constant energy barriers as represented by the symmetric factors $A(x, y)$, then the time-averaged current $J(x, y)$ along every transition $x \rightarrow y$ is zero. As a result, no net work can be done with such protocol.

The purpose of the paper is to extend the arguments proposed in Ref. 18 and to give the shortest general proof of this result (Sec. III), which at the same time also applies to classes of non-Markov models (Sec. IV) and for which the diffusion limit becomes equally simple (Sec. V). At the end we set the result into a broader context by showing that the time-dependent protocol under consideration in arbitrary (in general nonequilibrium) systems modifies all currents by a global multiplicative factor (Sec. VI). We start with the general setup in terms of a Markov jump process.

\section{SETUP}

Markov state models and their extensions are important tools for modeling thermodynamic processes of open systems ${ }^{19}$ and they find numerous applications in chemical kinetics ${ }^{19-22}$ and in biochemistry. ${ }^{23}$ In our abstract framework we use $x, y, \ldots$ to denote the long-lived or metastable states that locally minimize a given free energy landscape $G_{\alpha}(x)$ under equilibrium conditions; for instance, they refer to ch- 
emomechanical configuration of molecules within a suitable coarse-grained level of description. Together, they form the vertices of a stochastic network with bonds between pairs $(x y)$ indicating possible transitions. The dynamics is encoded in the transition rates $w_{\alpha}(x, y)$ satisfying the condition of detailed balance

$$
e^{-G_{\alpha}(x)} w_{\alpha}(x, y)=e^{-G_{\alpha}(y)} w_{\alpha}(y, x),
$$

with the free energies $G_{\alpha}$ in $k_{B} T$ units. The index $\alpha$ indicates a possible dependence on an externally controlled parameter which varies the depth of the local free energy minima $G_{\alpha}(x)$. Detailed balance (1) implies that for fixed $\alpha$, the dynamics (by assumption ergodic) relaxes to the equilibrium Gibbs distribution $\propto \exp \left[-G_{\alpha}(x)\right]$.

As indicated before, we restrict ourselves to a protocol that makes the transition rates,

$$
w_{t}(x, y)=A(x, y) e^{G_{\alpha(t)}(x)}, \quad A(x, y)=e^{-\Delta(x, y)},
$$

time dependent via the parameter $\alpha=\alpha(t)$ in the free energy minima $G_{\alpha} . \Delta(x, y)=\Delta(y, x)$ are the effective barrier heights which are kept fixed. In general, $A(x, y)$ just specifies the Arrhenius prefactor in the transition rates and $\Delta(x, y)$ deviates from a true barrier energy by geometrical corrections. However, a good approximation in many applications is to consider $G(x)$ and $\Delta(x, y)$ as independent in the sense that they can be manipulated independently from each other by suitably varying the minima and the barrier heights, respectively.

It has been shown in Ref. 16 that no energy pumping is possible for such systems in the following sense. Let $\rho_{t}$ be the instantaneous distribution function and let $j_{t}(x, y)$ be the corresponding instantaneous mean current between pairs of states,

$$
j_{t}(x, y)=\rho_{t}(x) w_{t}(x, y)-\rho_{t}(y) w_{t}(y, x),
$$

as obtained from the master equation written in the form

$$
\frac{d \rho_{t}(x)}{d t}+\sum_{y} j_{t}(x, y)=0 .
$$

There is no strictly stationary distribution as the rates are time dependent, but when the protocol $\alpha(t)$ is periodic in time, we expect to find that $\rho_{t}$ itself becomes periodic in time, at least for sufficiently large times $t$. In any event, we can define the time-averaged current

$$
J(x, y)=\lim _{T} \frac{1}{T} \int_{0}^{T} j_{t}(x, y) d t
$$

and the no-pumping theorem states that this long-time average equals zero for all pairs of states $x, y$. Thinking of independent particles hopping on the network over $x \rightarrow y$ with rate $w_{t}(x, y)$, no pumping refers to having no net timeaveraged flow of particles between any two nodes $x$ and $y$.

In the next section we present a simple derivation of this result within the present setup of Markov state models. Later sections will provide extensions to the semi-Markov and the diffusion systems, giving the main results of this paper.

\section{NO-PUMPING THEOREM}

Before starting with the proof we remind the reader of two important facts. First of all, the no-pumping theorem is only valid for some specific types of time dependence-in general, for those considered in Eq. (2). Even when the rates are satisfying the condition of detailed balance (1) for each fixed value of the parameter $\alpha$, there is no a priori reason why these could not arise a net current $J(x, y)$ in the process with time dependent $\alpha(t)$. In fact, that is exactly what happens in so-called flashing (and other) ratchets where the change in the potential landscape produces a net flow of particles. ${ }^{24}$ For example, a system (like a ratchet) with transition rates

$$
\widetilde{w}_{t}(x, y)=A(x, y) e^{-\left[G_{t}(y)-G_{t}(x)\right] / 2}, \quad A(x, y)=A(y, x)
$$

also satisfies detailed balance (1) for each fixed time $t$ and can be written analogous to Eq. (2) as

$$
\widetilde{w}_{t}(x, y)=e^{G_{t}(x)-\tilde{\Delta}_{t}(x, y)},
$$

but the effective barriers $\tilde{\Delta}_{t}(x, y)=\left[G_{t}(x)+G_{t}(y)\right] / 2$ $-\ln A(x, y)$ have become time dependent. Within the framework of the no-go theorem there is absolutely no reason now that the net currents would be identically zero (unless further symmetries are imposed).

Second, the geometry of the stochastic network is certainly relevant for the possible generation of a current. In fact, the net current $\int_{0}^{T} j_{t}(x, y) d t$ over any edge $(x y)$ connecting two otherwise disconnected subgraphs is a total time difference of the form $N_{T}(x, y)-N_{0}(x, y)$ and hence automatically approaches zero when time averaged as in Eq. (5). Thus $w_{t}(x, y)$ can be arbitrary (=no restriction) over such a "bridge" and the restricted form of time dependence as in Eq. (2) is only required over those edges $(x y)$ which belong to a loop.

We now come to our formulation of the no-pumping theorem. Consider the class of Markov jump processes with states $x, y, \ldots$ as in Sec. II. For all bonds $(x y)$ in our stochastic network that are part of a loop in the network, we require that the time dependence in the transition rates is of the form

$$
w_{t}(x, y)=\lambda_{t}(x) p(x, y),
$$

where $\lambda_{t}(x)=\Sigma_{y} w_{t}(x, y)$ is the time-dependent escape rate and $p(x, y)$ is a time-independent transition probability; $p(x, y) \geq 0, \Sigma_{y} p(x, y)=1$. We assume that the matrix $[p(x, y)]$ is irreducible so that there is a unique left eigenvector $\mu$ for eigenvalue 1: $\Sigma_{x} \mu(x) p(x, y)=\mu(y)$. (That is automatically so when the network of states is connected via $p(x, y)>0$-Perron-Frobenius theorem.) We also assume detailed balance, i.e., for some potential $V$,

$$
e^{-V(x)} p(x, y)=e^{-V(y)} p(y, x),
$$

so that in fact $\mu(x) \propto e^{-V(x)}$. Finally, we suppose that the limit

$$
\varpi(x)=\lim _{T} \frac{1}{T} \int_{0}^{T} \rho_{t}(x) \lambda_{t}(x) d t
$$

exists. (That is automatically satisfied when the time dependence is periodic but, clearly that is not strictly necessary.) 
The no-pumping theorem is now easily proven as follows. Since the full time evolution is obtained by solving Eqs. (3) and (4), the time integral of the latter gives

$$
0=\lim _{T} \frac{1}{T} \int_{0}^{T} \frac{d \rho_{t}}{d t} d t=-\sum_{y} J(x, y)
$$

and

$$
J(x, y)=\varpi(x) p(x, y)-\varpi(y) p(y, x),
$$

where we have inserted condition (6). Hence, $\Sigma_{x} \varpi(x) p(x, y)=\varpi(y)$, which is the stationary master equation for a time-independent Markov chain with (unnormalized) distribution $\varpi$ and current $J$. By irreducibility and by detailed balance (7), Eqs. (9) and (10) have the unique solution

$$
\varpi(x) \propto e^{-V(x)}, \quad J(x, y)=0 \quad \text { (all pairs) }
$$

as was to be proven.

Conditions (6) and (7) are just equivalent to Eqs. (1) and (2) and, hence, the above theorem can immediately be applied to the situation of Sec. II. Specifically, for a system with free energy wells $G_{\alpha}(x)$ and effective energy barriers $\Delta(x, y)=\Delta(y, x)$ as in Eq. (2), we consider an arbitrary cyclic path $\alpha(t)=\alpha(t+T)$ with some period $T$. Keeping the energy barriers constant, condition (6) is verified with timedependent escape rates

$$
\lambda_{t}(x)=e^{G_{\alpha(t)}(x)} \sum_{y} e^{-\Delta(x, y)}
$$

and time-independent transition probabilities

$$
p(x, y)=\frac{e^{-\Delta(x, y)}}{\sum_{y} e^{-\Delta(x, y)}},
$$

satisfying detailed balance (7) for $V(x)=-\ln \Sigma_{y} e^{-\Delta(x, y)}$.

As a summary, the essential ingredients are twofold. First, the original time-dependent jump process is detailed balanced for each fixed $t$, and second, the transition rates can be decomposed into a product of time-dependent escape rates $\lambda_{t}(x)$ and time-independent transition probabilities $p(x, y)$.

The idea of the proof above, at least for a periodic time dependence, is that the time-averaged current in the original system (2) exactly coincides with the stationary current in a temporally coarse-grained system with transition probabilities $p(x, y)$. As the original process is detailed balanced for each fixed time, the stationary coarse-grained process is time reversal symmetric and therefore the net current in the original process vanishes.

As a final comment, it is important to realize that the vanishing of the net (i.e., time-averaged) current, $J(x, y)=0$, does not imply that $j_{t}(x, y)=0$ at each time, unless the process runs in the quasistatic regime. Related to that, the overall dissipation does remain nonzero in general. For this it suffices to look at the time-averaged entropy flux (EF),

$$
\mathrm{EF} \equiv \frac{1}{2 T} \sum_{x, y} \int_{0}^{T} d t j_{t}(x, y) \ln \frac{w_{\alpha(t)}(x, y)}{w_{\alpha(t)}(y, x)} \geq 0 .
$$

We refer, e.g., to Sec. III in Ref. 25 for setting this expression for the EF in a thermodynamic context. Inserting Eq. (1) we compute

$$
\mathrm{EF}=\frac{1}{T} \int_{0}^{T} d t\left\langle\frac{d G_{\alpha(t)}}{d t}\right\rangle_{\rho_{t}}
$$

over the average work $\left\langle d G_{\alpha(t)} / d t\right\rangle_{\rho_{t}} \equiv \Sigma_{x} \rho_{t}(x) d G_{\alpha(t)}(x) / d t$. Only in the quasistatic (or adiabatic) limit when we can take $\rho_{t}(x) \propto \exp \left[-G_{\alpha(t)}(x)\right] \quad$ do we get that $\left\langle d G_{\alpha(t)} / d t\right\rangle_{\rho_{t}}$ $=-d / d t \ln \Sigma_{x} \exp \left[-G_{\alpha(t)}(x)\right]$ and is the $\mathrm{EF}=0$.

\section{NON-MARKOV GENERALIZATION}

By the simplicity of the proof above, the arguments allow a natural extension to more general jump processes including those with nonexponential waiting time distributions. These are called semi-Markov systems or continuous-time random walks, here on the chemical network. The fact that the presented method survives here is important, especially since many biophysical and biochemical processes are believed to be essentially non-Markovian for a natural choice of states. ${ }^{26}$

We consider a jump process for which the main change with respect to the Markov case consists in its dependence on the time $t_{0}$ of the previous jump. In that way, given that the system is in state $x$ at time $t$ since its last jump to $x$ at $t_{0}$, the probability that the next jump occurs within the time interval $[t, t+d t]$ is given by $\lambda\left(x ; t_{0}, t\right) d t$. [The Markov case corresponds to $\lambda\left(x ; t_{0}, t\right)=\lambda_{t}(x)$.] The probability rate that the next jump goes to $y$ is then

$$
w\left(x, t_{0} ; y, t\right)=\lambda\left(x ; t_{0}, t\right) p(x, y)
$$

generalizing the time-dependent Markov transition rate (6). We keep the same assumptions on the transition matrix $[p(x, y)]$, with its most important property being the condition of detailed balance (7). The $p(x, y)$ define what is often called the embedded Markov chain. The complication of the memory present in the escape rates $\lambda\left(x ; t_{0}, t\right)$ turns out to be irrelevant for our proof of the no pumping, as we now show.

The probability density that at time $t$ the system is found in state $x$ and that the last jump before $t$ occurred within $\left[t_{0}, t_{0}+d t_{0}\right]$ is denoted by $\rho\left(x ; t_{0}, t\right) d t_{0}$-it relates to the standard single-time distribution as

$$
\rho_{t}(x)=\int_{0}^{t} \rho\left(x ; t_{0}, t\right) d t_{0} .
$$

The mean current $j\left(x, t_{0} ; y, t\right) d t_{0}$ counts the expected rate of (directed) jumps $x \rightleftarrows y$ at time $t$ when the previous jump occurred in $\left[t_{0}, t_{0}+d t_{0}\right]$,

$$
j\left(x, t_{0} ; y, t\right)=\rho\left(x ; t_{0}, t\right) w\left(x, t_{0} ; y, t\right)-\rho\left(y ; t_{0}, t\right) w\left(y, t_{0} ; x, t\right) .
$$

It is related to the standard mean current as 


$$
j_{t}(x, y)=\int_{0}^{t} j\left(x, t_{0} ; y, t\right) d t_{0} .
$$

By construction, the single-time quantities $\rho_{t}$ and $j_{t}$ satisfy the balance equation

$$
\frac{d \rho_{t}(x)}{d t}+\sum_{y} j_{t}(x, y)=0
$$

We proceed analogously as in the Markov case. We assume that the limiting quantities

$$
\begin{aligned}
& \varpi(x)=\lim _{T} \frac{1}{T} \int_{0}^{T} \int_{0}^{t} \rho\left(x ; t_{0}, t\right) \lambda\left(x ; t_{0}, t\right) d t_{0} d t, \\
& J(x, y)=\lim _{T} \frac{1}{T} \int_{0}^{T} j_{t}(x, y) d t
\end{aligned}
$$

are well defined. Then again,

$$
J(x, y)=\varpi(x) p(x, y)-\varpi(y) p(y, x)
$$

and from integrating Eq. (15),

$$
\sum_{y} J(x, y)=0
$$

By detailed balance (7) we reach the conclusion $J(x, y)=0$ which ends the proof. As before, time homogeneity and detailed balance of the embedded Markov chain imply that the net flux through any pair (xy) asymptotically goes to zero.

\section{DIFFUSION LIMIT}

The same ideas apply to diffusion processes. We can obtain them as continuum limits of Markov jump processes. For simplicity we assume here that the stochastic network is a one-dimensional chain, possibly turned into a circle. The lattice spacing is denoted by $\epsilon$ and we will take the continuum limit $\epsilon \rightarrow 0$. We think of the transitions as the hopping of a random walker between nearest neighbor sites $x$ $\rightarrow x \pm \epsilon$ with rates depending on space- and time-dependent amplitudes $D_{t}(x)$ and a potential $U_{t}(x)$,

$$
\begin{aligned}
w^{\epsilon}(x, x \pm \epsilon) & =\epsilon^{-2} \sqrt{D_{t}(x) D_{t}(x \pm \epsilon)} e^{\left[U_{t}(x)-U_{t}(x \pm \epsilon)\right] / 2} \\
& =\epsilon^{-2} D_{t}(x) \pm \frac{1}{2} \epsilon^{-1} D_{t}(x)\left[\ln D_{t}-U_{t}\right]^{\prime}(x)+\mathcal{O}(1),
\end{aligned}
$$

by expanding around $\epsilon=0$ and with $\mathcal{O}(1)$ indicating an error term that remains bounded along $\epsilon \rightarrow 0$. As in Eq. (6), we decompose these transition rates $w^{\epsilon}(x, x \pm \epsilon)$ into a product of escape rates $\lambda_{t}^{\epsilon}(x)$ and transition probabilities $p^{\epsilon}(x, x \pm \epsilon)$, to find

$$
\lambda_{t}^{\epsilon}(x)=2 \epsilon^{-2} D_{t}(x)+\mathcal{O}(1)
$$

and

$$
\begin{aligned}
p_{\epsilon}(x, x \pm \epsilon) & =\frac{1}{2} \pm \frac{\epsilon}{4}\left[\ln D_{t}-U_{t}\right]^{\prime}(x)+\mathcal{O}(1) \\
& =\frac{1}{2} e^{[V(x)-V(x \pm \epsilon)] / 2}+\mathcal{O}(1)
\end{aligned}
$$

under the condition that $V=U_{t}-\ln D_{t}$ does not depend on time. That then reproduces form (6) under which the no pumping holds. This comparison to jump processes predicts under what natural condition we may expect zero net current also for diffusions. Next we give a direct argument that confirms this idea.

We are now dealing with a Langevin-type equation in Itô form

$$
d x_{t}=-D_{t}\left(x_{t}\right) U_{t}^{\prime}\left(x_{t}\right) d t+D_{t}^{\prime}\left(x_{t}\right) d t+\sqrt{2 D_{t}\left(x_{t}\right)} d B_{t},
$$

where $d B_{t} / d t$ indicates standard white noise. That describes an overdamped one-dimensional diffusion on a ring (for periodic boundary condition) or on the line, in a timedependent potential landscape $U_{t}(x)$ and with timedependent diffusion parameter $D_{t}(x)$ (setting the environment temperature equal to one).

The corresponding Fokker-Planck equation reads

$$
\begin{aligned}
& \frac{\partial \rho_{t}(x)}{\partial t}+j_{t}^{\prime}(x)=0, \\
& j_{t}(x)=-\rho_{t}(x) D_{t}(x) U^{\prime}(x)-D_{t}(x) \rho_{t}^{\prime}(x) .
\end{aligned}
$$

The same ideas as above can now be applied. As before we define the quantities

$$
\varpi(x)=\lim _{T} \frac{1}{T} \int_{0}^{T} \rho_{t}(x) D_{t}(x) d t
$$

and the time-averaged current

$$
J(x)=\lim _{T} \frac{1}{T} \int_{0}^{T} j_{t}(x) d t .
$$

By time integrating Eq. (22) we reach the stationarity condition

$$
J^{\prime}(x)=0 .
$$

For "effective" potential $V=U_{t}-\ln D_{t}$ and assuming that $V$ is time independent, one gets

$$
\begin{aligned}
J(x) & =\lim _{T} \frac{1}{T} \int_{0}^{T}\left[-\rho_{t} D_{t} V^{\prime}(x)-\left(\rho_{t} D_{t}\right)^{\prime}\right] d t \\
& =-\varpi(x) V^{\prime}(x)-\varpi^{\prime}(x) .
\end{aligned}
$$

As a consequence, the time-averaged characteristics of the original process (21) coincide with the stationary characteristics of a time-independent detailed balanced diffusion in the potential landscape $V$ with unit diffusion parameter. Therefore, $\varpi(x) \propto \exp [-V(x)]$ and $J=0$ is the (unique) solution.

We conclude that for diffusion process with only gradient forces the condition, 


$$
\frac{\partial}{\partial t}\left(D_{t} e^{-U_{t}}\right)=0
$$

is sufficient for the vanishing of the average current (24). We recognize in Eq. (27) the condition for time independence of the transition probability $p^{\epsilon}(x, x \pm \epsilon)$ of the random walk in Eq. (21). Our result also agrees with the no-pumping condition found previously by a more complicated method. ${ }^{17} \mathrm{~A}$ no-pumping theorem for a general Langevin process on a compact manifold of arbitrary dimension was given in Ref. 18 , using a method similar to the above.

\section{NONEQUILIBRIUM GENERALIZATION}

A natural question arises how the extra time-dependence affecting only the energy wells or escape rates such as in Eq. (6) modifies the long-time characteristics of a general nonequilibrium system with steady currents already present. We answer here that question by generalizing the above argument, restricting ourselves to the case of jump processes (Sec. III). This will throw more light into the nature and robustness of no-pumping theorems.

As before in Eq. (2) we start from transition rates

$$
w_{t}(x, y)=w(x, y) e^{G_{t}(x)},
$$

with time-dependent energy function $G_{t}(x)=G_{\alpha(t)}(x)$ but now we do not assume detailed balance for the reference process with rate $w(x, y)$. That reference could thus very well correspond to a driven nonequilibrium but time-homogeneous system. Let $\rho^{s}(x)$ be the stationary density of the reference, with steady currents $j^{s}(x, y)=w(x, y) \rho^{s}(x)-\rho^{s}(y) w(y, x)$ and $\Sigma_{y} j^{s}(x, y)=0$.

Inspecting formulas (9)-(11), we find that the long timeaveraged current is

$$
J(x, y)=\lim _{T} \frac{1}{T} \int_{0}^{T} j_{t}(x, y) d t=\varpi(x) w(x, y)-\varpi(y) w(y, x),
$$

with

$$
\varpi(x)=\lim _{T} \frac{1}{T} \int_{0}^{T} \rho_{t}(x) e^{G_{t}(x)} d t
$$

and satisfying the stationarity condition in all nodes

$$
\sum_{y} J(x, y)=0
$$

By the assumed ergodicity, Eqs. (29) and (30) have a unique solution in the form

$$
\varpi(x)=\Omega \rho^{s}(x), \quad J(x, y)=\Omega j^{s}(x, y),
$$

with the normalization

$$
\Omega=\lim _{T} \frac{1}{T} \sum_{x} \int_{0}^{T} \rho_{t}(x) e^{G_{t}(x)} d t
$$

Hence, we have arrived at an important conclusion: For the time-dependent protocols under consideration, the timeaverage current (29) is merely a global multiplicative factor of the reference steady current. If the latter is zero, there is also no resulting pumped current and we recover the original results.

Remark that the ergodicity assumption is not essential: in general, the dynamics decomposes into several ergodic components made of mutually connected states. Within each of the components, the reference stationary distribution $\rho^{s}$ is unique and the above argument readily applies, up to that the current multiplicative factor $\Omega$ is now generally different for each component and it depends on the initial distribution $\rho_{0}$.

In the case of a quasistatic process the system passes through the states

$$
\rho_{t}(x)=\frac{1}{Z_{t}} \rho^{s}(x) e^{-G_{t}(x)}, \quad Z_{t}=\sum_{x} \rho^{s}(x) e^{-G_{t}(x)},
$$

which are stationary with respect to the instantaneous energy landscape $G_{t}$. Indeed, these distributions correspond to the currents $j_{t}(x, y)=j^{s}(x, y) / Z_{t}$ such that $\Sigma_{y} j_{t}(x, y)=0$. The normalization factor (32) then becomes

$$
\Omega=\lim _{T} \frac{1}{T} \int_{0}^{T} \frac{d t}{Z_{t}}
$$

One also checks that the total dissipation as measured by the time-integrated outgoing entropy flux (12) gets modified by the same factor $\Omega$ (but only) in the adiabatic limit.

\section{CONCLUSION}

The no-pumping theorem relies on the decomposition of the jump rates into a time-dependent escape rate and a timehomogeneous stochastic matrix (embedded Markov chain). Then, time-integrated currents depend on the given timedependent protocol only via a global multiplicative factor. When the embedded Markov chain satisfies detailed balance, the no-pumping result appears.

While the temporal coarse graining can indeed express the original time-integrated current in terms of the steady current for the embedded Markov chain, that is not true for the dissipation or entropy flux which remains nonzero even in case the embedded Markov chain is detailed balanced. The same ideas and methods of proof apply to semi-Markov processes and to the diffusion limit.

\section{ACKNOWLEDGMENTS}

The authors thank Chris Jarzynski for initial discussions. C.M. benefits from the Belgian Interuniversity Attraction Poles Programme P6/02. K.N. acknowledges the support from the Academy of Sciences of the Czech Republic under Project No. AV0Z10100520 and from the Grant Agency of the Czech Republic under Grant No. 202/07/J051. This work was started by C.M. and K.N. at the Yukawa Institute for Theoretical Physics in Kyoto, while attending the YKIS 2009 "Frontiers in Nonequilibrium Physics."

\footnotetext{
${ }^{1}$ T. Y. Tsong, J. Biol. Phys. 28, 309 (2002).

${ }^{2}$ W. R. Browne and B. L. Feringa, Nat. Nanotechnol. 1, 25 (2006).

${ }^{3}$ Molecular Motors, edited by M. Schliwa (Wiley-VCH, New York, 2003).

${ }^{4}$ R. D. Vale and R. A. Milligan, Science 288, 88 (2000).

${ }^{5}$ D. A. Leigh, J. K. Y. Wong, F. Dehez, and F. Zerbetto, Nature (London) 424, 174 (2003).

${ }^{6}$ J. V. Hernández, E. R. Kay, and D. A. Leigh, Science 306, 1532 (2004).
} 
${ }^{7}$ M. Klok, N. Boyle, M. T. Pryce, A. Meetsma, W. R. Browne, and B. L. Feringa, J. Am. Chem. Soc. 130, 10484 (2008).

${ }^{8}$ T. R. Kelly, H. De Silva, and R. A. Silva, Nature (London) 401, 150 (1999).

${ }^{9}$ N. Koumura, R. W. J. Zijlstra, R. A. Delden, N. Harada, and B. L. Feringa, Nature (London) 401, 152 (1999).

${ }^{10}$ D. J. Thouless, Phys. Rev. B 27, 6083 (1983).

${ }^{11}$ R. D. Astumian and I. Derényi, Phys. Rev. Lett. 86, 3859 (2001).

${ }^{12}$ R. D. Astumian, Phys. Rev. Lett. 91, 118102 (2003).

${ }^{13}$ R. D. Astumian, Proc. Natl. Acad. Sci. U.S.A. 104, 19715 (2007).

${ }^{14}$ N. A. Sinitsyn and I. Nemenman, Phys. Rev. Lett. 99, 220408 (2007).

${ }^{15}$ V. Y. Chernyak and N. A. Sinitsyn, J. Chem. Phys. 131, 181101 (2009).

${ }^{16}$ S. Rahav, J. Horowitz, and C. Jarzynski, Phys. Rev. Lett. 101, 140602 (2008).

${ }^{17}$ J. Horowitz and C. Jarzynski, J. Stat. Phys. 136, 917 (2009).
${ }^{18}$ V. Y. Chernyak and N. A. Sinitsyn, Phys. Rev. Lett. 101, 160601 (2008).

${ }^{19}$ J. Keizer, Statistical Thermodynamics of Nonequilibrium Processes (Springer-Verlag, Berlin, 1987).

${ }^{20}$ S. Park and V. S. Pande, J. Chem. Phys. 124, 054118 (2006).

${ }^{21}$ S. P. Elmer, S. Park, and V. S. Pande, J. Chem. Phys. 123, 114903 (2005).

${ }^{22}$ G. R. Bowman, K. A. Beauchamp, G. Boxer, and V. S. Pande, J. Chem. Phys. 131, 124101 (2009).

${ }^{23}$ M. Kurzynski, The Thermodynamic Machinery of Life (Springer-Verlag, Berlin, 2006).

${ }^{24}$ P. Reimann, Phys. Rep. 361, 57 (2002).

${ }^{25}$ C. Maes, K. Netočný, and B. Wynants, Markov Processes Relat. Fields 14, 445 (2008).

${ }^{26}$ A. Fuliński, Z. Grzywna, I. Mellor, Z. Siwy, and P. N. R. Usherwood, Phys. Rev. E 58, 919 (1998). 